\title{
Foreword
}

\section{International Workshop on Materials Design Process: Thermodynamics, Kinetics and Microstructure Control}

The discovery, development, and deployment of new materials involve much knowledge and incorporation of thermodynamics, kinetics, and microstructure control. Therefore, forming a materials design/control process integrates computational, experimental, and data informatics tools and in turn enables accelerating the development of advanced materials. In order to intensify interactions and collaborations in the areas, the International Workshop on Materials Design Process: Thermodynamics, Kinetics and Microstructure Control (TKM-2013) was successfully held at the IMDEA Materials Institute, Getafe, Madrid, Spain (June 3 to 4, 2013).

Subjected to the standard peer-review procedure in Metallurgical and Materials Transactions A, a total of eight papers were accepted for publishing in the current issue. The selected papers, starting with a review in phase-field modeling of polycrystalline solidification, principally appear in order consistently with the conference topics, i.e., thermodynamics, kinetics, microstructure, and applications in design/control. We are very grateful to all the conference participants (particularly the keynote speakers) for their excellent contributions, Ms. Dora Moscatello and Prof. David E. Laughlin for their kind approval of the symposium publication, and all the referees for reviewing the papers.

Symposium Organizers:

Yuwen Cui, Staff Researcher

IMDEA Materials Institute, Madrid, Spain yuwen.cui@imdea.org

Jose M. Torralba, Professor IMDEA Materials Institute, Madrid, Spain Universidad Carlos III Madrid, Madrid, Spain

Xiao-Gang Lu, Professor Shanghai University, Shanghai, P.R. China 